\title{
Psychiatric symptoms as the first manifestation of juvenile SLE complicated with Klinefelter's syndrome
}

\author{
Mehrnoush Hassas Yeganeh", Reza Shiari \\ From 21st European Pediatric Rheumatology (PReS) Congress \\ Belgrade, Serbia. 17-21 September 2014
}

\section{Introduction}

Systemic Lupus Erythematosus (SLE) is an autoimmune, multisystem disorder with diverse manifestations. There are limited reports on the neuropsychiatric findings as the first manifestation of systemic lupus erythematosus in children.

\section{Objectives}

A 14-year-old Iranian boy with two years history of cognitive dysfunction, behavioural problems and recent history of epistaxis was referred to Mofid's Children Hospital, Tehran, Iran. His work-up ended to a diagnosis of Klinefelter's syndrome associated with juvenile systemic lupus erythematosus.

\section{Methods}

It is a case report. It just reports a patient with unusual manifestation of systemic lupus erythematosus. The patient also had a chromosomal abnormality discovered by chromosomal study and the diagnosis of Klinefelter's syndrome was confirmed.

\section{Results}

Patients with Klinefelter's syndrome may exhibit behavioural problems and psychological distress. These psychiatric disorders will be more prominent if be complicated with Lupus in children. In fact, psychiatric symptoms can occur as the first manifestation of juvenile SLE.

\section{Conclusion}

In pediatric patients with psychiatric disorders, especially in those who do not respond well to the classic

Paediatric Rheumatology, Children's Hospital, Tehran, Iran, Islamic Republic Of 